\title{
Inactivation of Protein Tyrosine Phosphatases by Peracids Correlates with the Hydrocarbon Chain Length
}

\author{
Alicja Kuban-Jankowska ${ }^{a}$ Magdalena Gorska Jack A. Tuszynskib,c \\ Cassandra D. M. Churchilld Philip Winter ${ }^{c}$ Mariusz Klobukowskid \\ Michal Wozniak ${ }^{\mathrm{a}}$
}

aDepartment of Medical Chemistry, Medical University of Gdansk, Gdansk, Poland; b Department of Physics, University of Alberta, Edmonton, Canada; 'Department of Oncology, University of Alberta, Edmonton, Canada; ${ }^{d}$ Department of Chemistry, University of Alberta, Edmonton, Canada

\section{Key Words}

Protein tyrosine phosphatases (PTPs) • Peracids (peroxyacids, percarboxylic acids) • Catalytic cysteine residue

\begin{abstract}
Background/Aims: Protein tyrosine phosphatases are crucial enzymes controlling numerous physiological and pathophysiological events and can be regulated by oxidation of the catalytic domain cysteine residue. Peracids are highly oxidizing compounds, and thus may induce inactivation of PTPs. The aim of the present study was to evaluate the inhibitory effect of peracids with different length of hydrocarbon chain on the activity of selected PTPs. Methods: The enzymatic activity of human CD45, PTP1B, LAR, bacterial YopH was assayed under the cell-free conditions, and activity of cellular CD45 in human Jurkat cell lysates. The molecular docking and molecular dynamics were performed to evaluate the peracids binding to the CD45 active site. Results: Here we demonstrate that peracids reduce enzymatic activity of recombinant CD45, PTP1B, LAR, YopH and cellular CD45. Our studies indicate that peracids are more potent inhibitors of CD45 than hydrogen peroxide (with an IC50 value equal to $25 \mathrm{nM}$ for peroctanoic acid and $8 \mu \mathrm{M}$ for hydrogen peroxide). The experimental data show that the inactivation caused by peracids is dependent on hydrocarbon chain length of peracids with maximum inhibitory effect of medium-chain peracids (C8-C12 acyl chain), which correlates with calculated binding affinities to the CD45 active site. Conclusion: Peracids are potent inhibitors of PTPs with the strongest inhibitory effect observed for medium-chain peracids.
\end{abstract}

\section{Introduction}

Copyright (c) 2015 S. Karger AG, Basel

Protein tyrosine phosphatases (PTPs) dephosphorylate phosphotyrosine residues in proteins. Together with protein tyrosine kinases (PTKs), PTPs are responsible for the

Dr. Alicja Kuban-Jankowska

KARGER 125
Department of Medical Chemistry, Medical University of Gdansk, Debinki Street 1, 80210 Gdansk (Poland)

Tel. +48 5834914 50, E-Mail alicjakuban@gumed.edu.pl 
regulation of the tyrosine phosphorylation status, which controls numerous cellular processes, such as cellular growth, differentiation, metabolism, cell-cell communication and immune response. The balance between opposing activities of PTPs and PTKs is fundamental for maintaining homeostasis, and any disturbance of this balance may contribute to disease development [1].

The hallmark defining the classical PTP enzymes is the strictly-conserved active site sequence $\mathrm{C}(\mathrm{X})_{5} \mathrm{R}$ within the catalytic domain, which constitutes the phosphate-binding pocket of the enzyme [2]. The cysteine residue inside the signature motif participates directly in the removal of phosphate groups from certain phosphoproteins. The cysteine residue located at the bottom of the active site cleft exists in the thiolate anion form, and is highly prone to oxidation [3]. Oxidation of the cysteine residue to sulfenic acid residue leads to phosphatase inactivation but is reversible and re-conversion to cysteine residue can be provided by cellular reducing agents. However a highly oxidizing environment can induce further and irreversible oxidation, yielding sulfinic and sulfonic acid residues (Fig. 1A), both of which cause inactivation of the enzyme [4]. Oxidative stress, defined as excessive reactive oxygen species formation, may induce inactivation of protein tyrosine phosphatases. Inactivation via oxidation was suggested as a mechanism of PTPs regulation [5].

One of the key representatives of receptor-like PTPs is CD45, which controls many cellular processes. CD45 dephosphorylates, among others, the Src-family kinases, and thus is a key positive regulator of $\mathrm{T}$ or B cell receptor-mediated signaling leading to lymphocyte activation [6]. Moreover, CD45 plays an important role in allergic response, being required in mast cells for the degranulation process and subsequent histamine release [7]. In pancreatic acinar cells, CD45 was found to operate as a negative regulator of the cytokine receptor signaling pathway via suppression of JAK kinases [8]. Phosphatase CD45 was also reported to be implicated in carcinogenesis since over-expression of the CD45 encoding gene correlates with an increased proliferation rate of myeloma cells [9].

Human leukocyte antigen-related (LAR) phosphatase is a receptor-like transmembrane phosphatase expressed in brain tissue. LAR-phosphatases have recently emerged as a major family of synaptic adhesion molecules [10]. LAR acts as a negative regulator of multiple receptor tyrosine kinases [11].

Phosphatase PTP1B is localized to the cytoplasmic face of the endoplasmic reticulum and is expressed ubiquitously, including in the classical insulin-targeted tissues such as liver, muscle and fat. Mounting evidence from biochemical, genetic and pharmacological studies supports a role for PTP1B as a negative regulator in both insulin and leptin signaling. PTP1B can associate with and dephosphorylate an activated insulin receptor (IR) or insulin receptor substrates (IRS) [12]. There is evidence that phosphatase PTP1B is involved in pathogenesis of type 2 diabetes and obesity [13]. PTP1B is a target in breast cancer treatment [14], but it can also play the role of a tumor suppressor [15].

Fig. 1. Reactions of PTPs inactivation, peracids formation and decomposition. (A) Inactivation of PTPs by oxidation of cysteine residue in the active site to reversible form of sulfenic acid or irreversible form of sulfinic and sulfonic acid residues. (B) Peroxyacetic acid formation from acetic acid and hydrogen peroxide. (C) Decomposition of peroxyacetic acid to acetic acid and oxygen.

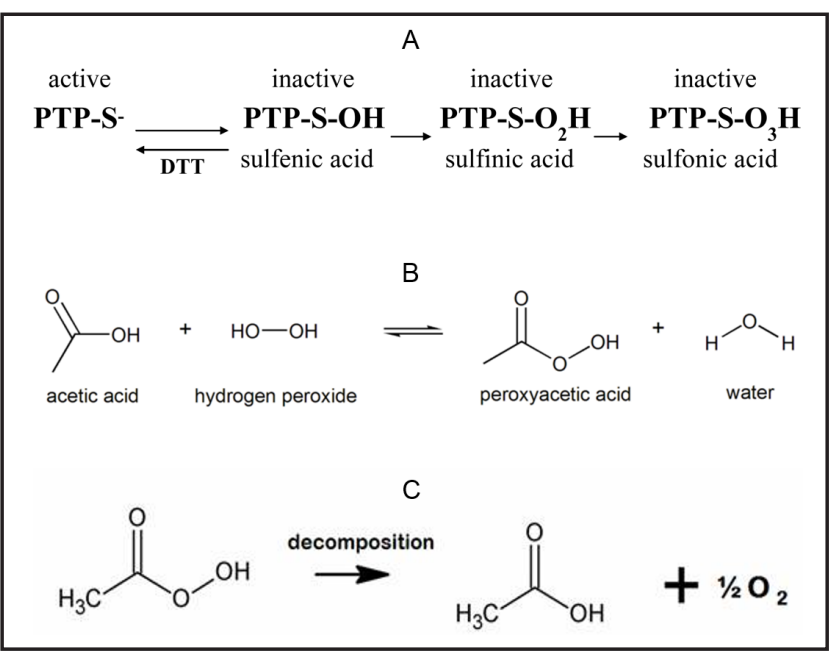


Protein tyrosine phosphatase YopH is expressed in "Yersinia" species bacteria as virulence effector and plays a role of bacterial agency, negatively controlling signaling pathways of a host cell required for phagocytosis [16]. YopH is one of the most active protein tyrosine phosphatases characterized to date [17].

Hydrogen peroxide is known to inhibit protein tyrosine phosphatases via oxidation of the catalytic cysteine [4]. Moreover, hydrogen peroxide in the presence of carboxylic acids may lead to the formation of the respective peroxycarboxylic acids (peracids) [18] (Fig. 1B). Peracids are highly reactive (Fig. 1C) and are therefore more potent oxidants than hydrogen peroxide [19]. It has been shown that peracetic acid (C2-peracid), likely formed in chronic alcoholics from acetic acid and hydrogen peroxide, may inhibit enzyme that contains thiol groups, arachidonoyl-CoA: 1-palmitoyl-sn-glycero-3-phosphocholine acyl transferase, localized in the erythrocyte membrane [20]. Another study has shown that peracetic acid (C2-peracid) is a potent oxidative inhibitor of PTP1B phosphatase [21]. In our previous work, we demonstrated that activity of phosphatase CD 45 can be decreased by treatment with tetradecanoic peracid [22]. Interestingly, the active site of PTPs is highly positively charged and may be an attractor for negatively charged peroxycarboxylic group of peracids [23, 24].

The aim of the present study was the assessment of the inhibitory effect of selected peracids on the enzymatic activity of recombinant CD45, PTP1B, LAR and CD45 phosphatase in Jurkat cells. We used peracids with different hydrocarbon chain length to estimate the peracid with the most inhibitory effect. This was compared to the inhibitory impact of hydrogen peroxide.

\section{Materials and Methods}

\section{Cell line and cell culture}

The human Jurkat T cell line, clone E.6-1, was obtained from European Collection of Cell Culture (ECACC, UK). The cells were cultured at $37^{\circ} \mathrm{C}$ in RPMI 1640 medium supplemented with $10 \%$ fetal bovine serum, $100 \mu \mathrm{g} / \mathrm{mL}$ penicillin/streptomycin and $2 \mathrm{mM}$ L-glutamine. The culture was maintained at $37^{\circ} \mathrm{C}$ and in an atmosphere containing 5\% $\mathrm{CO}_{2}$. RPMI 1640 medium and supplements were obtained from SigmaAldrich. The cell culture density was kept at $1 \times 10^{6}$ cells $/ \mathrm{mL}$. At least every two days the medium was replaced with the fresh one, and the cells were counted and reseeded to maintain the recommended density. Concentration of protein in Jurkat cell lysate was measured using the Bradford colorimetric method. The Bradford method is based on Coomassie Brilliant Blue G-250 absorbance shift in the presence of protein. Binding to the protein being assayed under acidic conditions, the red dye is converted into the blue derivative. The amount of protein in the sample is proportional to the amount of bound dye, and thus to increase of an absorbance at $595 \mathrm{~nm}$. Based on prepared standard concentrations of bovine serum albumin, concentration of protein in samples was calculated.

\section{Cell viability assay with MTT}

The Jurkat cells $\left(1 \times 10^{6}\right.$ cells $\left./ \mathrm{mL}\right)$ untreated (control) or treated with solution of hydrogen peroxide or peracids after the appropriate incubation time were suspended in solution of $0.5 \mathrm{mg} / \mathrm{mL}$ MTT(3-[4,5dimethylthiazol-2-yl]-2,5-diphenyltetrazolium bromide) in RPMI 1640 without phenol red. The $100 \mu \mathrm{L}$ samples were incubated for $2-4$ hours at $37^{\circ} \mathrm{C}$ in 96 -well plates. When the purple precipitate was clearly visible under the microscope, $100 \mu \mathrm{L}$ of DMSO was added to each well and the plate with cover was left in the dark for 2-4 hours. The absorbance at $540 \mathrm{~nm}$ was determined using a microplate reader.

\section{Determination of PTP CD45 activity in cell lysate}

The Jurkat cells (density at $1 \times 10^{6}$ cells $/ \mathrm{mL}$ ) were untreated (control) or treated with solution of hydrogen peroxide or peracids and incubated for 24 hours at $37^{\circ} \mathrm{C}$ in 24 -well plates. The cells were rinsed twice with TBS, suspended at the density of 1 x $10^{7}$ cells/mL in Lysis buffer pH 7.4 with 0.5 \% NP-40, 25 gg/ mL leupeptin, $25 \mu \mathrm{g} / \mathrm{mL}$ pepstatin, $2 \mu \mathrm{g} / \mathrm{mL}$ aprotinin, $1 \mathrm{mM}$ PMSF, vortexed briefly and placed on ice for 15 minutes. The cells were then solubilized by forcing the lysates through a 19-gauge needle $0.686 \mathrm{~mm}$ inner diameter) 20 times and centrifuged at $12000 \mathrm{x}$ g at $4^{\circ} \mathrm{C}$ for 5 minutes. The supernatants were transferred 


\section{Cellular Physiology Cell Physiol Biochem 2015;36:1069-1083 \begin{tabular}{ll|l} 
and Biochemistry & $\begin{array}{l}\text { DOI: 10.1159/000430280 } \\
\text { Published online: June 18, } 2015\end{array}$ & $\begin{array}{l}\text { O 2015 S. Karger AG, Basel } \\
\text { www.karger.com/cpb }\end{array}$ \\
\cline { 2 - 3 }
\end{tabular}}

Kuban-Jankowska et al.: PTPs as Targets for Peracids

to test tubes and assayed immediately. The day prior to the assay, the 96-well microplates were coated with CD45 capture antibodies ( $8 \mu \mathrm{g} / \mathrm{mL}$ in PBS) and incubated overnight at room temperature. After washing the wells, cell lysate was added, and the plate was placed on a rocking platform at $30 \mathrm{rpm}$ for 3 hours at room temperature. Lysates were aspirated from the wells and PTP activity was measured colorimetrically using $200 \mu \mathrm{M}$ tyrosine phosphate substrate (phosphopeptide DADEY(PO3)LIPQQG in $10 \mathrm{mM}$ HEPES buffer pH 7.4) and malachite green. The phosphopeptide substrate was dephosphorylated by active CD45 to generate unphosphorylated peptide and free phosphate. The free phosphate was then detected by a sensitive dye binding assay using malachite green and molybdic acid. The increase in absorbance at $620 \mathrm{~nm}$ was measured with the microplate reader. The activity of CD45 was determined by calculating the rate of phosphate release. CD45 capture antibody, tyrosine phosphate substrate DADEY(P03)LIPQQG, malachite green and molybdic acid were purchased from R\&D Systems. Detergent NP-40, protease inhibitors (leupeptin, pepstatin, aprotinin) and phenylmethylsulfonylfluoride (PMSF) were purchased from Sigma-Aldrich.

\section{Recombinant PTP CD45, PTP1B, YopH and LAR activity assay}

Human recombinant $\mathrm{CD} 45$ and bacterial recombinant YopH protein tyrosine phosphatase were obtained from Sigma-Aldrich, PTP1B from Prospec and LAR phosphatase from Calbiochem. The solutions of the recombinant PTPs was prepared in $10 \mathrm{mM}$ HEPES buffer $\mathrm{pH}$ 7.4. The final concentration of phosphatase in reaction samples was $0.8 \mu \mathrm{g} / \mathrm{mL}(10 \mathrm{nM})$. The CD45, PTP1B, LAR and YopH enzymes were untreated (control) or treated with solution of hydrogen peroxide and selected peracids. The assay was performed in 96-well microplates, and the final volume of each sample was $200 \mu \mathrm{L}$. The enzymatic activities of CD45, PTP1B, LAR, YopH were measured using 1mM chromogenic substrate para-nitrophenyl phosphate ( $p$ NPP) in $10 \mathrm{mM}$ HEPES buffer $\mathrm{pH} 7.4$, at $37^{\circ} \mathrm{C}$. Phosphatase hydrolyzed $p$ NPP to para-nitrophenol and inorganic phosphate. Para-nitrophenol is an intensely yellow colored soluble product under alkaline conditions. The increase in absorbance (due to para-nitrophenol formation) is linearly proportional to enzymic activity concentration (with excessive substrate, i.e. zero-order kinetics) and was assessed at $405 \mathrm{~nm}$ on a microplate reader Jupiter (Biogenet) using DigiRead Communication Software (Asys Hitech GmbH).

Subsequently, recombinant phosphatase CD45 that had been previously inactivated by selected peracids or hydrogen peroxide, was then treated with $10 \mathrm{mM}$ dithiothreitol (DTT), and the samples were incubated for 15 minutes at $37^{\circ} \mathrm{C}$ to reverse the inactivation, if possible. Restoration of CD45 enzymatic activity was measured as an increase of absorbance taken at $405 \mathrm{~nm}$ as previously described. The amount of modified CD45 thiol adduct with NBD (Cys-S-NBD adduct) was measured after 30 min incubation with $\mathrm{NDB}-\mathrm{Cl}(0.6 \mathrm{mM}$ in a $0.5 \mathrm{~mL}$ of sample) as absorbance at $420 \mathrm{~nm}$ with spectrophotometer.

\section{Peracid synthesis}

Peracids were synthesized according Parker's method [25] as a result of the reaction of a carboxylic acid of interest, and hydrogen peroxide. The carboxylic acid of interest was added into the solution of $50-60 \%$ hydrogen peroxide and 70\% sulfuric acid (as a reaction medium), and the reaction mixture was incubated for 2 hours at a room temperature, and then purified. The identity and purity of the synthesized peracids were tested with NMR and IR spectroscopy. Peroxy acids were deep frozen and stored in a powder form at $-80^{\circ} \mathrm{C}$. Unfortunately, due to highly explosive properties of short-chain peracids during synthesis process, we have not obtained the C4 and C6-peracid.

\section{Molecular docking}

AutoDockTools version 1.5.4 was used to convert the receptor and ligand library to PDBQT format [26]. The molecular docking program AutoDock Vina version 1.1.1 was used to perform the docking [27]. The preparation of the enzyme model is described elsewhere [22]. The catalytic domain of CD45 was used to model the enzyme, with Cys853 in an anionic state and Asp821 in a neutral state, as required for catalysis [28]. A binding box was defined centered on the position of the phosphorus atom in the phosphotyrosine peptide in the bound complex. The largest ligand in a fully extended conformation was C16; the longest distance between atom coordinates for this ligand was calculated to be about $25 \AA$. The binding box for docking had to be large enough to contain this ligand, therefore the binding box was set at a cube with the side length of $25 \AA$ A. Docking with a fully rigid receptor was performed. The Vina parameter exhaustiveness increases the time spent on the search; for the final docking runs an exhaustiveness parameter of 128 was 
used. Preliminary docking runs suggested that this parameter was more than sufficient; increasing the exhaustiveness is not expected to significantly change the results for the rigid docking. A set of 6 repetitions were performed with different random seeds. Since the search of ligand performed by Vina is stochastic, the results will be different depending on the random seed. The reported binding affinities are the means of all the repetitions, and the reported errors are the standard deviations. The best binding pose was defined as the pose with the strongest affinity in the largest cluster of poses, with poses clustered with a $1.5 \AA$ RMSD thresholds.

\section{Molecular dynamics}

Molecular dynamics simulations were performed to obtain refined free energies of binding and structural information. Simulations were performed for dianionic phosphotyrosine, while the C4, C8 and C12 peracids, having $\mathrm{pK}$ 's in the range of 8.1-10.8 [19], were modeled in both neutral and anionic forms. The structures obtained by molecular docking were used as input for the molecular dynamics simulations. Ligand parameters for the GAFF forcefield [29] were derived with the Antechamber module of AmberTools. Protein components were described with the Amber ff99SB forcefield [30]. Each enzyme-ligand complex was solvated in a TIP3P octahedral water box extending $21 \AA$ from the solute. Sodium and chloride ions were added to neutralize the system and obtain a physiologically-relevant ion concentration of $0.1 \mathrm{M}$. Nonbonded terms were calculated within a 10 Å cutoff, while long-range electrostatics were calculated with the particle-mesh Ewald. During simulations, the SHAKE algorithm was utilized.

Using Amber 12 with GPUs [31-34], the solvated system was simulated using the following procedure: With the protein-ligand complex under strong restraints, 2000 cycles of steepest descent and 1000 cycles of conjugate gradient minimizations were performed. With restraints removed, 4000 cycles of steepest descent and 2000 steps of conjugate gradient minimizations were performed. The system was initially heated from $0 \mathrm{~K}$ to $100 \mathrm{~K}$ for 20 ps using a $1 \mathrm{fs}$ timestep at constant NVT with weak restraints on solute. Subsequently, heating was performed from $100 \mathrm{~K}$ to $300 \mathrm{~K}$ for $100 \mathrm{ps}$ with a 1 fs timestep at constant NPT with weak restraints on solute. Next, restraints were removed for 100 ps with a 1 fs timestep at constant NPT at $300 \mathrm{~K}$.

Following the above preparations of the system, a final run was performed at constant NVT at $300 \mathrm{~K}$ for $15 \mathrm{~ns}$ with a $2 \mathrm{fs}$ timestep from which production data were collected. The final $12.5 \mathrm{~ns}$ of this simulation was analyzed. To calculate the free energy of binding, the Molecular Mechanics/Generalized Born Surface Area (MM/GBSA) method was used. The GBn formulation [35] was implemented along with newly-developed parameters [36] and mbondi3 radii. The entropic component of binding was evaluated with the normal mode approximation. The reported binding free energies are averaged over the course of the final $12.5 \mathrm{~ns}$ of the simulation (Fig. 6B). Pairwise-binding contributions reported between the ligand and residues only contain non-entropic components of the binding free energy (Fig. 6C).

\section{Statistical analysis}

The experiments were performed at least three times. The data were applied and analyzed with GraphPad Prism (GraphPad Software v.4). Statistical analyses were performed using ANOVA combined with Tukey's test or T test combined with Wilcoxon test. The data were expressed as means \pm SD. Differences between means were considered significant for $\mathrm{P}<0.05$.

\section{Results}

Peracids are more potent inhibitors of CD45 than hydrogen peroxide

In the first step of the study, we determined the inhibitory effect of hydrogen peroxide (Fig. 2A) and selected peracids (Fig. 2B,C) on enzymatic activity of recombinant CD45 phosphatase. The results obtained in CD45 activity assay show that peracids inhibit CD45 phosphatase more strongly than hydrogen peroxide by several orders of magnitude, with an IC50 value equal to $25 \mathrm{nM}$ for peroctanoic acid and $40 \mathrm{nM}$ for perdecanoic acid, as compared to $8 \mu \mathrm{M}$ for hydrogen peroxide (Fig. 2C).

Recombinant protein tyrosine phosphatase CD45 was used to identify the effect of hydrogen peroxide on its enzymatic activity. CD45 was treated with various concentrations of hydrogen peroxide for 15 minutes and the percent of retained enzymatic activity of CD45 


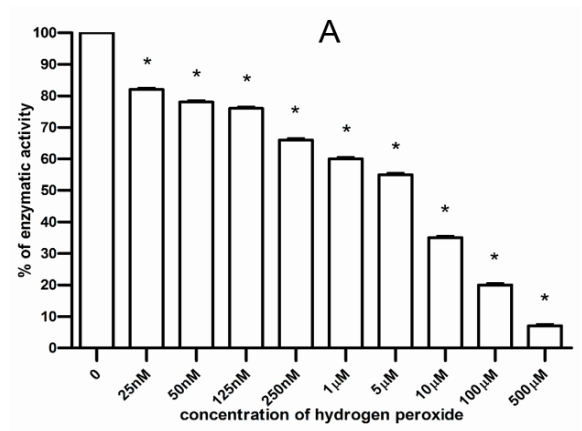

C

\begin{tabular}{|l|l|l|}
\hline Name of inhibitor & Chemical formula of inhibitor & IC 50 value \\
\hline Peracetic acid (C2) & $\mathrm{CH}_{3} \mathrm{COOOH}$ & $5 \mu \mathrm{M}$ \\
\hline Peroctanoic acid (C8) & $\mathrm{CH}_{3}\left(\mathrm{CH}_{2}\right)_{6} \mathrm{COOOH}$ & $25 \mathrm{nM}$ \\
\hline Perdecanoic acid (C10) & $\mathrm{CH}_{3}\left(\mathrm{CH}_{2}\right)_{8} \mathrm{COOOH}$ & $40 \mathrm{nM}$ \\
\hline Perdodecanoic acid (C12) & $\mathrm{CH}_{3}\left(\mathrm{CH}_{2}\right)_{10} \mathrm{COOOH}$ & $41 \mathrm{nM}$ \\
\hline Pertetradecanoic acid $(\mathrm{C} 14)$ & $\mathrm{CH}_{3}\left(\mathrm{CH}_{2}\right)_{12} \mathrm{COOOH}$ & $53 \mathrm{nM}$ \\
\hline Hexadecanoic acid $(\mathrm{C} 16)$ & $\mathrm{CH}_{3}\left(\mathrm{CH}_{2}\right)_{14} \mathrm{COOOH}$ & $140 \mathrm{nM}$ \\
\hline Hydrogen peroxide & $\mathrm{H}_{2} \mathrm{O}_{2}$ & $8 \mu \mathrm{M}$ \\
\hline
\end{tabular}

B

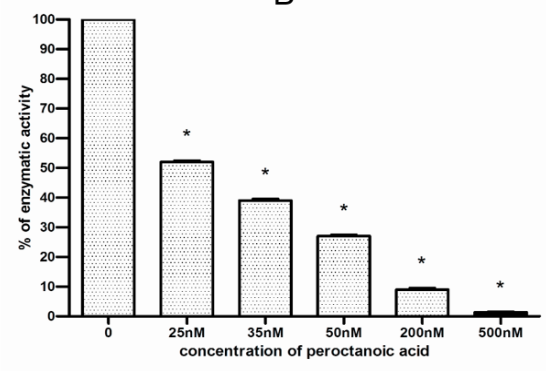

$\mathrm{D}$

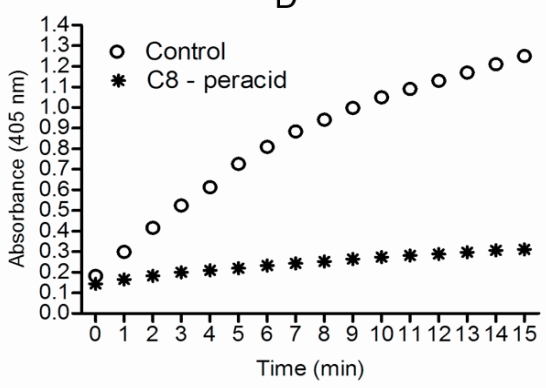

Fig. 2. Recombinant CD45 activity assay with hydrogen peroxide and selected peracids as inhibitors. (A) Impact of hydrogen peroxide on enzymatic activity of recombinant protein tyrosine phosphatase CD45. Data presented as means \pm SD $(n=10)$. One-way Anova combined with Tukey test. ${ }^{*}$ significantly different $(\mathrm{P}<0.001)$ from control. (B) Impact of various concentrations of peroctanoic acid on enzymatic activity of recombinant phosphatase CD45. Data presented as means \pm SD $(n=10)$. One-way Anova test. * significantly different $(\mathrm{P}<0.001)$ from control. (C) $\mathrm{IC}_{50}$ values of peracids and hydrogen peroxide for CD45 inhibition. $\mathrm{IC}_{50}$ values were determined from a plot presenting peracid or hydrogen peroxide concentration versus percentage of the enzymatic activity of recombinant CD45 after 15 minutes incubation with inhibitors. (D) Activity of recombinant CD45 after treatment with $50 \mathrm{nM}$ C8-peracid in comparison to control. The activity was measured every 1 minute as an absorbance with $p$ NPP substrate.

was assessed (Fig. 2A). A small effect was observed for $25 \mathrm{nM}$ hydrogen peroxide, which resulted in a reduction in CD45 enzymatic activity to nearly $80 \%$. A major loss in activity, to less than $10 \%$, was observed after the treatment of CD45 with $500 \mu \mathrm{M}$ hydrogen peroxide (Fig. 2A).

In a subsequent experiment, recombinant protein tyrosine phosphatase CD45 was treated with different concentrations of peroctanoic acid. The percent of retained enzymatic activity of CD45, following 15 minutes of incubation with peroctanoic acid, was measured (Fig. 2B). Peroctanoic acid with a concentration of $25 \mathrm{nM}$ reduced CD45 enzymatic activity to $50 \%$, as compared to the control. Nearly complete loss of activity of CD45 (activity less than $2 \%$ ) was observed after treatment with $500 \mathrm{nM}$ peroctanoic acid (Fig. 2B). The loss of CD45 activity as a function of time after treatment with $50 \mathrm{nM}$ peroctanoic acid in comparison to a control is shown in Figure 2D.

Molecular docking calculations show that peracids have a higher binding affinity than hydrogen peroxide for the CD45 active site (with binding affinities of $-5.7 \mathrm{kcal} / \mathrm{mol}$ for C8peracid, in comparison to $-3.1 \mathrm{kcal} / \mathrm{mol}$ for hydrogen peroxide). This is consistent with the experimental results obtained using the recombinant phosphatase, and may explain the greater inhibitory impact of peracids on CD45 as compared to hydrogen peroxide (Fig. 2). 
Fig. 3. Cellular CD45 activity and viability assay. (A) Inhibitory effect of peracids $(50 \mu \mathrm{M})$ of different hydrocarbon chain length or hydrogen peroxide $(50 \mu \mathrm{M})$ on CD45 enzymatic activity in Jurkat cells. Cells were treated with peracids or hydrogen peroxide and then lysed. Activity of CD45 was measured using immunoprecipitation technique, and the specific substrate. Data presented as mean \pm SD $(n=3)$. One-way analysis of variance. * significantly different $(\mathrm{P}<0.001)$ from control. (B) Cell viability determined with MTT assay. Data presented as the percentage of living cells after treatment with $50 \mu \mathrm{M}$ peracids or $50 \mu \mathrm{M}$ hydrogen peroxide for 24 hours.
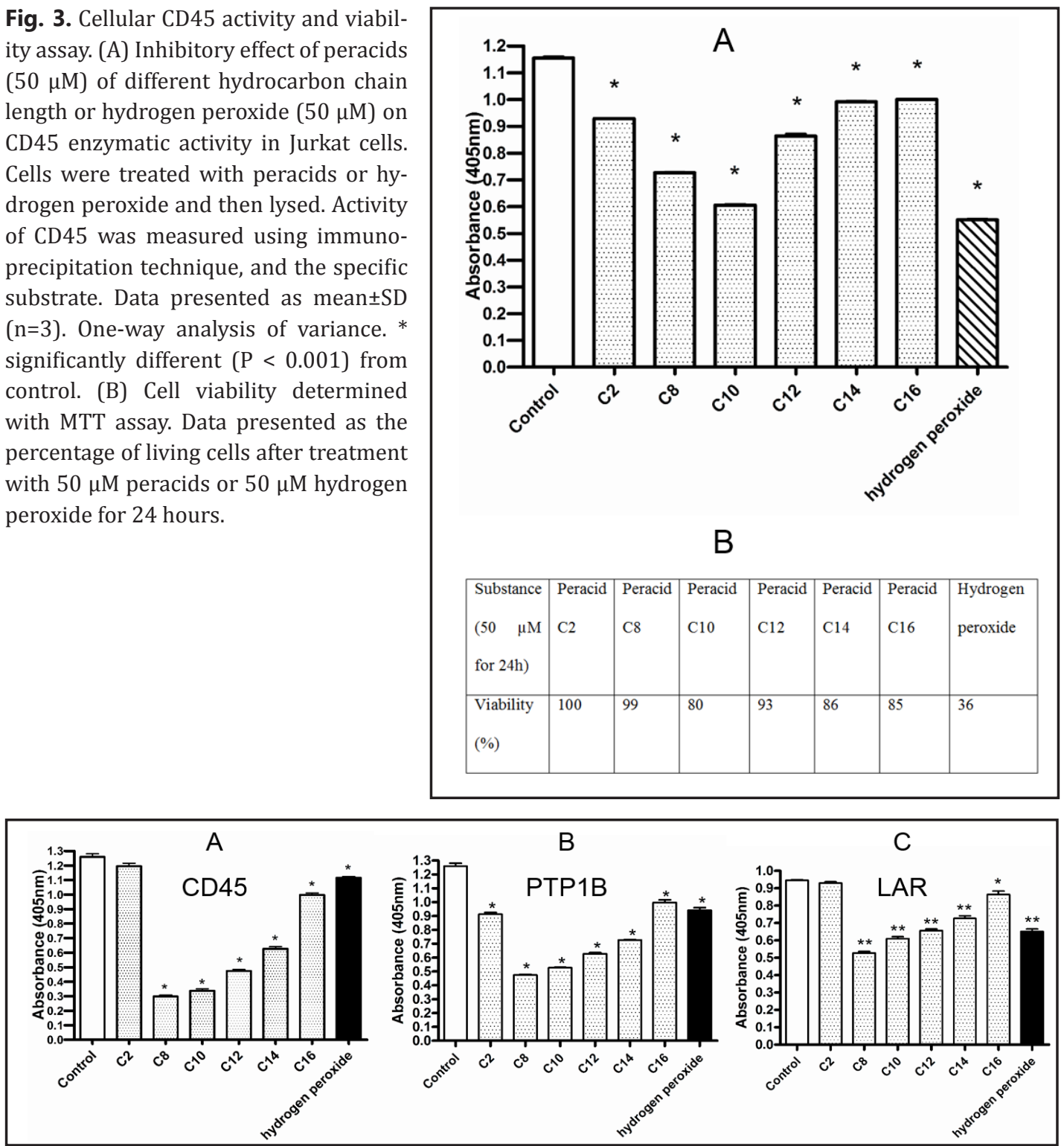

Fig. 4. Recombinant CD45, PTP1B and LAR activity assay. (A) Impact of peracids (50 nM) of different hydrocarbon chain length or hydrogen peroxide $(50 \mathrm{nM})$ on the enzymatic activity of recombinant CD 45 . Data presented as mean \pm SD $(n=10)$. One-way analysis of variance. * significantly different $(P<0.001)$ from control. (B) Impact of different length hydrocarbonyl chain peracids (50 $\mathrm{nM})$ and hydrogen peroxide (50 $\mathrm{nM})$ on the enzymatic activity of PTP1B. Data presented as mean \pm SD $(n=10)$. One-way analysis of variance. * significantly different $(\mathrm{P}<0.001)$ from control. (C) Impact of different length hydrocarbonyl chain peracids $(50 \mathrm{nM})$ and hydrogen peroxide $(50 \mathrm{nM})$ on the enzymatic activity of LAR. Data presents mean $\pm S D(n=10)$. One-way analysis of variance. ${ }^{*}$ significantly different $(\mathrm{P}<0.01)$ from control. ${ }^{* *}$ significantly different $(\mathrm{P}<0.001)$ from control.

\section{Peracids induce inactivation of CD45 in Jurkat cells}

We found that peracids are able to reduce enzymatic activity of the cellular CD45 present in Jurkat cells. In this experiment, Jurkat cells were treated with $50 \mu \mathrm{M}$ of peracids of differing acyl chain lengths (including 2, 8, 10,12, 14 and 16 carbons), or hydrogen peroxide, at room temperature for 1 hour. The enzymatic activity of CD 45 was assessed in Jurkat cell lysates (Fig. 3A). Perdecanoic acid (C10) was observed to have the strongest inhibitory effect 


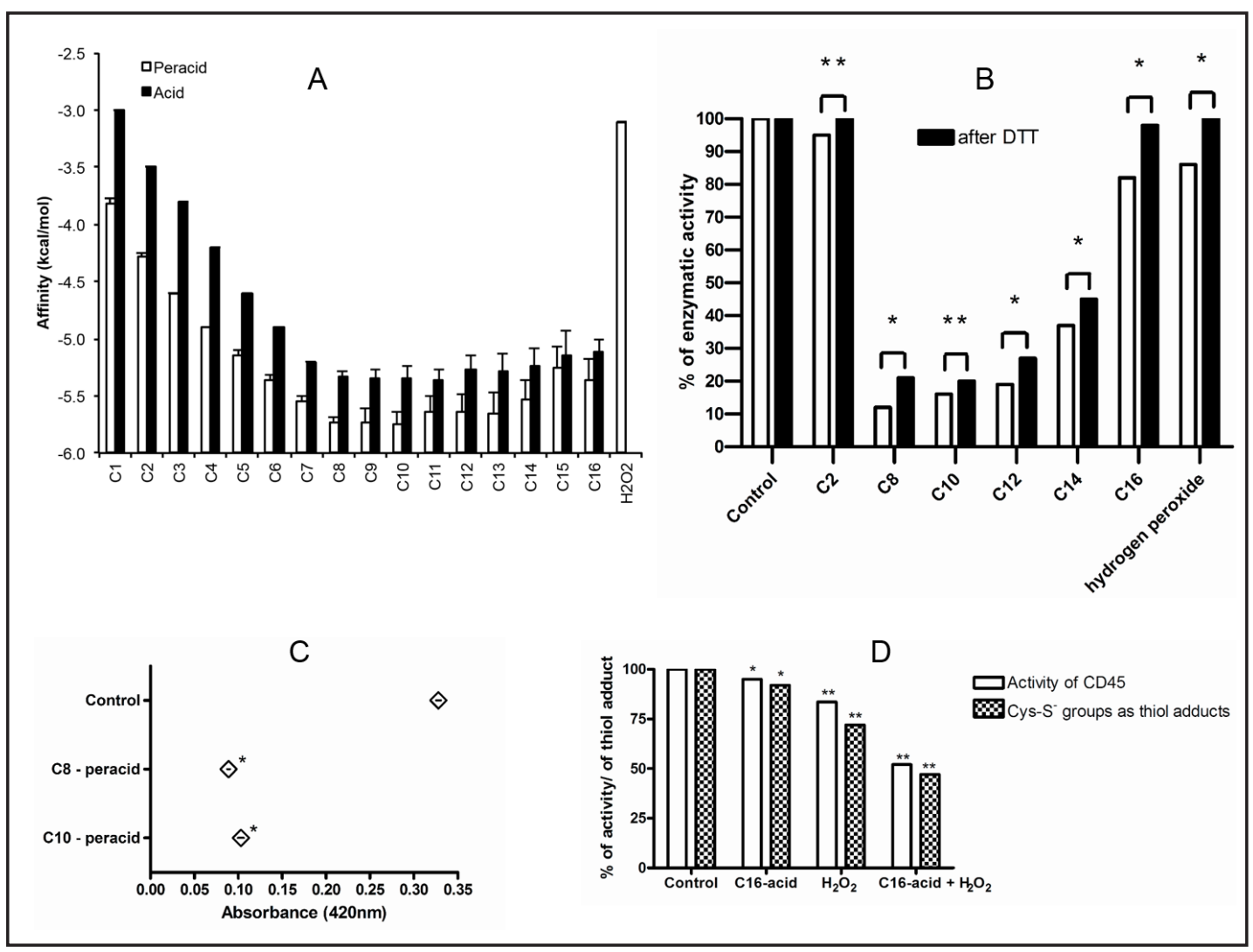

Fig. 5. Mechanism of peracids induced CD45 inactivation studies. (A) The calculated binding affinities (in kcal mol-1) of peracids of different acyl chain length (C1-C16), their corresponding carboxylic acids, and hydrogen peroxide. The ligands were docked in a binding box centered on the phosphatase active site of a 3D structural model of the D1 domain of human CD45. The binding affinities were calculated with AutoDock Vina with flexible ligands and a rigid receptor. The error bars are standard deviations of 6 repetitions with different random seeds. (B) The restoration of CD45 activity with DTT. Inactivation of CD45 by selected peracids $(50 \mathrm{nM})$ or hydrogen peroxide $(50 \mathrm{nM})$, and the subsequent reversibility of the inactivation after treatment with $10 \mathrm{mM}$ DTT. Data presented as mean \pm SD $(n=10)$. T-test analysis of variance. * significantly different $(\mathrm{P}<0.05)$ in pairs, ${ }^{* *}$ significantly different $(\mathrm{P}<0.01)$ in pairs. $(\mathrm{C})$ The amount of modified CD45 thiol adduct with NBD (Cys-S-NBD adduct) after treatment with C8 and C10 peracids. Data presented as absorbance at $420 \mathrm{~nm}$. ${ }^{*}$ significantly different $(\mathrm{P}<0.001$ ) from control. (D) Activity of CD45 and the amount of modified CD45 thiol adduct with NBD after treatment with C16- acid, $\mathrm{H}_{2} \mathrm{O}_{2}$ or C16- acid with $\mathrm{H}_{2} \mathrm{O}_{2}$. Data presented as a percent of control, mean $\pm \mathrm{SD}(\mathrm{n}=3)$. ${ }^{*}$ significantly different $(\mathrm{P}<0.01)$ from control, ${ }^{* *}$ significantly different from control $(\mathrm{P}<0.001)$.

on CD45 enzymatic activity, while the inhibitory effect decreased with both longer or shorter acyl chain lengths of the peracids.

A cell viability analysis showed that treatment of Jurkat cells with $50 \mu \mathrm{M}$ peracids for 24 hours has little no impact on cell viability, while incubation of Jurkat cells with the same concentration of hydrogen peroxide induces 64\% loss of viability (Fig. 3B). Data are presented as the percentage of living cells compared to control (100\%).

\section{Medium-chain peracids are stronger inhibitors of PTPS}

The results obtained in the next experiments showed that peracids with different number of carbons in the hydrocarbon chain inactivate PTPs with diverse effects. Inhibition was found to be related to the length of the acyl chain of the peracid. The medium-chain length peracids exhibited the highest impact on the enzymatic activity of CD45, PTP1B and 
LAR phosphatases (Fig. 4). The overall trend of the inactivation relative to the length of the hydrocarbon chain of peracids was the same for each phosphatase tested.

Recombinant CD45, PTP1B and LAR phosphatase were treated with $50 \mathrm{nM}$ peracids of varying acyl chain lengths or with hydrogen peroxide. The obtained results showed that peroctanoic acid (C8-peracid) has the greatest impact on the enzymatic activity of CD45, PTP1B and LAR phosphatases (Fig. 4). The inhibitory effect decreased for peracids with longer acyl chains. The peracetic acid containing 2 carbons was found to have less inhibitory impact on CD45, PTP1B and LAR than the medium chain peracids (Fig. 4).

\section{Mechanism of peracids induced inactivation of CD45}

The dependency of the inhibitory effect on acyl chain length was observed for both the recombinant CD45 (Fig. 2C,4A) and CD45 natively expressed in Jurkat cells (Fig. 3A). Out of the investigated peracids, peroctanoic acid (with 8 carbons in the acyl chain) proved to be the most potent inhibitor of the recombinant CD45, while perdecanoic acid (with 10 carbons in the chain) proved to have the strongest inhibitory effect on cellular CD45. The inhibitory effect decreased both with an increasing or decreasing number of carbon atoms in the peracid hydrocarbon chain. This dependency on chain length allows us to hypothesize that medium-chain peracids have the optimal size and structure to effectively penetrate into the catalytic center of CD 45 .

In order to study the mechanism of CD45 inactivation by different peracids or hydrogen peroxide, the potential reversibility of the inhibitory effect was tested (Fig. 5B). In this study, recombinant tyrosine phosphatase CD45 was used to investigate the potential restoration of the enzymatic activity after inactivation hydrogen peroxide or peracids induced inactivation. CD45 that had been previously inactivated by hydrogen peroxide or peracids was subsequently incubated with $10 \mathrm{mM}$ dithiotreitol (DTT) to reverse the inactivation, if possible. The restoration of enzymatic activity of CD45 is presented in Figure 5A. We observed that the inactivation of CD45 by hydrogen peroxide was completely reversed by treatment with DTT. Similarly, the inactivation caused by the shortest chain (C2) as well as longest chain (C16) peracids was also found to be reversible. However, the inactivation of CD45 by medium-chain peracids (C8-C14) was only slightly restored by DTT, resulting in a maximum activity of $40 \%$ of the original activity.

The conclusion that medium-chain peracids induce conversion of cysteine residue to sulfinic and sulfonic acid residues is supported by the results obtained in NBD-Cl studies. We tested the amount of thiol groups modified by NBD-Cl forming Cys-S-NBD adducts. After treatment of recombinant CD 45 with the C8- or C10-peracid, the quantity of Cys-S-NBD adducts was significantly decreased in comparison to control (Fig. 5C). C8-peracid led to a $73 \%$ reduction and $\mathrm{C} 10$-peracid to a $67 \%$ reduction of thiol-NBD adducts, suggesting that thiol groups in CD45 after treatment with selected peracids are in oxidized forms.

Interestingly, in the course of the study we observed an additional inhibitory effect of palmitic acid (C16-acid) and hydrogen peroxide on the enzymatic activity of recombinant CD45. The inhibitory effect of C16-acid was rather low (5\%), whereas in presence of hydrogen peroxide it was significantly enhanced and inhibited $50 \%$ of CD 45 activity, which is also much stronger than the effect of hydrogen peroxide alone (less than 20\%) (Fig. 5D). The obtained results raised the possibility that the carboxylic acid may become activated by hydrogen peroxide to form peracids.

Peracids may bind within the active site of PTPS

We performed molecular docking experiments for a series of peracids, and the results showed that peracids were not sterically precluded from binding in the catalytic center of CD45. Figure 6A shows the most plausible binding pose within the CD45 active site for C8-peracid. In this binding conformation, the peroxycarboxyl group of peroctanoic acid is a relatively short distance away from the catalytic cysteine residue (Cys853) $(<4 \AA$ ) and the arginine residue (Arg859) $(<2 \AA)$. Under such steric conditions there is a likelihood of hydrogen bond formation between the arginine residue and the peroxycarboxyl group. 


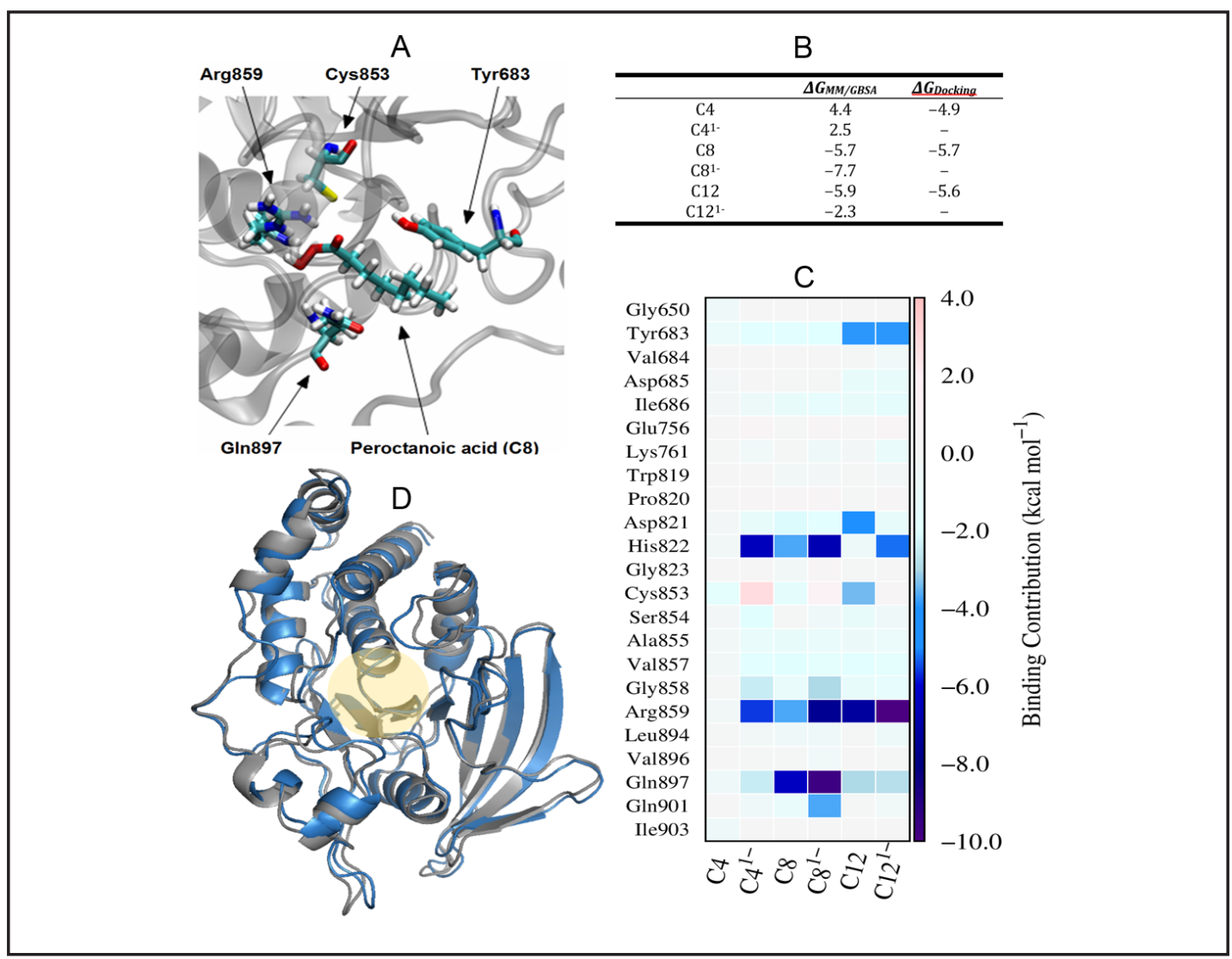

Fig. 6. Molecular docking and molecular dynamics studies of peracids binding to CD45 phosphatase active site. (A) The most thermodynamically plausible ligand binding poses for peroctanoic acid (C8) as determined by docking experiments performed with AutoDock Vina using a rigid receptor and a binding box centered on the CD45 phosphatase active site. The CD45 binding site backbone is shown as a grey cartoon and four amino acid residues critical for the binding are shown in stick form (the residue positions are numbered according to the UniProtKB sequence P08575). (B) The binding free energy of selected peracids to CD45 determined by molecular dynamics using the MM/GBSA method $\left(\Delta G_{M M / G B S A}\right.$ ), and the binding affinity determined by molecular docking ( $\Delta G_{\text {Docking }}$; see Fig 5 a). For the MM/GBSA computations, the energies were computed with both neutral forms and anionic forms $\left({ }^{1-}\right.$ ); for the docking computations, only neutral forms were used. Values are in $\mathrm{kcal} \mathrm{mol}^{-1}$. (C) The pairwise decomposition of the non-entropic component of the MM/GBSA binding free energy between CD45 active-site residues and selected peracids $\left(\mathrm{kcal} \mathrm{mol}^{-1}\right.$, with the strongest binding in blue). (D) An overlay of the enzyme-ligand complex (atom RMSD = $1.330 \AA$ ) for bound phosphotyrosine (blue) and C ${ }^{1-}$ (grey) showcasing the structural similarity of the enzyme in the presence of substrate (phosphotyrosine) or inhibitor (peroctanoic acid, C8). The active site region is highlighted in yellow. Structures are taken from the end of the 15 ns simulations.

The relatively short distance between the acyl chain and the tyrosine residue (Tyr683) $(<4$ $\AA$ A) should lead to hydrophobic interactions between the acyl chain and the tyrosine side chain. These interactions contributed to the relatively strong calculated binding affinity of peroctanoic acid.

The examination of the binding pose for C8-peracid (Fig. 6A) also shows, that in the predicted best binding pose, the peroxycarboxyl group of the peracid is directed toward the catalytic cysteine residue. This conformation would allow for the reaction between the peroxycarboxyl group and the cysteine residue, resulting in the oxidation of the cysteine residue.

The molecular docking studies found that peracids have a higher binding affinity to the CD45 active site than hydrogen peroxide, with binding affinity $-5.7 \mathrm{kcal} / \mathrm{mol}$ for C8- 


\section{Cellular Physiology Cell Physiol Biochem 2015;36:1069-1083 \begin{tabular}{l|l} 
and Biochemistry Published online: June 18, 2015 & $\begin{array}{l}\text { C } 2015 \text { S. Karger AG, Basel } \\
\text { www.karger.com/cpb }\end{array}$ \\
\hline
\end{tabular} \\ Kuban-Jankowska et al.: PTPs as Targets for Peracids}

peracid, in comparison to $-3.1 \mathrm{kcal} / \mathrm{mol}$ for hydrogen peroxide. This is consistent with the experimental results obtained using the recombinant phosphatase, and may explain the greater inhibitory impact of peracids on CD45 as compared to hydrogen peroxide (Fig. 2C). Furthermore, the computational analysis found that medium-chain peracids bind most strongly to the CD45 active site, with the maximum binding affinity for the C10-peracid (Fig. $5 \mathrm{~A}$ ). The docking studies provide support to the hypothesis that the observed differences in the inhibitory effects on CD45 activity are due to differences in binding to the CD45 active site.

Interestingly, the calculated binding affinities of the peracids as compared with the respective affinities for carboxylic acids are stronger, but the overall binding affinity trend as dependent on the number of carbon atoms in an the acyl chain is comparable for both the peracids and the carboxylic acids. Due to the fact that nanomolar concentrations of carboxylic acids have only a slightly inhibitory effect on CD 45 activity, we concluded that the peroxycarboxyl group is directly implicated in the mechanism of enzymatic inhibition, and is more important for inhibitory activity than the length of the acyl chain.

\section{Molecular dynamics studies of inhibitor binding}

To refine the results of the docking studies, molecular dynamics simulations were performed. The binding enthalpy of select peracids (C4-, C8- and C12-peracids) was calculated (Fig. 6B) and decomposed into per-residue pairwise contributions (Fig. 6C). Since peracids may be either neutral $(\mathrm{C} 4, \mathrm{C} 8, \mathrm{C} 12)$ or anionic $\left(\mathrm{C}^{1-}, \mathrm{C} 8^{1-}, \mathrm{C} 12^{1-}\right)$ under physiological conditions, they were modeled in both these forms. During the simulation of the neutral C4-peracid (denoted C4), the peracid dissociated away from the CD45 active site, which is reflected in the low affinity (4.4 $\left.\mathrm{kcal} \mathrm{mol}^{-1}\right)$ for this ligand for the binding site (Fig. 6B). All other peracids remained in the active site with varying affinities (Fig. 6B). The strongest attractive interactions with the C4-, C8- and C12-peracids were observed mainly for Arg859 and Gln897. Strong binding enthalpies are also observed with His822 for with the anionic peracids. Overall, the peracids exhibit a greater binding enthalpy in their anionic form.

A structural comparison was made to evaluate whether allosteric structural changes in the enzyme play a role in the inhibition of CD 45 by peracids, using either a phosphotyrosinebound complex or a peracid-bound complex. For each of the ligands, an average structure was obtained over the course of the final 15 ns of a molecular dynamics simulation. An alignment of the backbone heavy atoms was generated for natural substrate, phosphotyrosine, (Fig. 6D, blue) and the anionic C8-peracid (Fig. 6D, grey). These structures are very similar, having an atom RMSD of $1.330 \AA$.

Within the active site, minimal changes are observed in the enzyme structure in the presence of the substrate (phosphotyrosine) or inhibitors (C4-, C8-, C12-peracid) (Fig. 6D). A small deviation is seen in an active-site loop, where the side chains of Asp821, His822 and Gly823 deviate in the presence of the natural substrate and inhibitor (Fig. 6D, yellow). Specifically, hydrogen bonding with Asp821 is no longer present for the C8 ${ }^{1-}$ inhibitor. The results of this overlay indicate that structural changes in the enzyme are largely removed from the active site and likely do not influence the binding of the substrate or inhibitors.

\section{Discussion}

Peracids are a class of highly oxidizing compounds, and thus may induce inactivation of PTPs via oxidation of the catalytic cysteine residue. It had previously been found that peroxidized arachidonic acid can induce oxidation of PTPs [37]. Another study has shown that peracetic acid is a potent oxidative inhibitor of PTP1B phosphatase [21]. The peroxycarboxyl group $(-\mathrm{COOOH})$ in a peracid is an oxidized derivative of the regular carboxyl group $(-\mathrm{COOH})$ [18]. Peracids can be produced as a result of the reaction between carboxylic acids and hydrogen peroxide (Fig. 1B), e.g. peracetic acid will be formed in the reaction of hydrogen peroxide with acetic acid. Hydrogen peroxide activation, with no metal ions involved, also leads to peracid generation. In this mechanism, the activation of hydrogen peroxide is caused 
by perhydrolysis of a carbonyl precursor [38]. This reaction can occur spontaneously or can be catalyzed by certain enzymes. It was demonstrated that lipases catalyzed the synthesis of tetradecanoic peracid (C14-peracid) from hydrogen peroxide and tetradecanoic acid [39].

Hydrogen peroxide is naturally produced in various organisms (mitochondrial respiratory chain, oxidases, and specifically in the metabolism of white blood cells), and subsequently hydrogen peroxide can convert carboxylic acids $(-\mathrm{COOH})$ into peracids $(-\mathrm{COOOH})$. The risk of peracid formation corresponds to increased production of carboxylic acids. Such over-synthesis of carboxylic acids has been reported in some liver disorders and psoriaris [40, 41]. High amounts of free carboxylic acid, such as palmitic or stearic acids are generated in type 2 diabetes [42]. In addition, recent studies demonstrate an inhibitory effect of free fatty acids on PTP1B and SHP2 phosphatase [43, 44].

Peracids can undergo decomposition to carboxylic acids, simultaneously releasing active oxygen (Fig. 1C) [19]. The stability of peracids in aqueous solution is $\mathrm{pH}$ dependent. Peracids may be decomposed at alkaline pHs. The mechanism of peracid decomposition results in the formation of highly reactive singlet molecular oxygen [19].

The experiments we performed demonstrate that nanomolar concentrations of peracids can inactivate human recombinant phosphatases CD45, PTP1B and LAR (Fig. 2,4). Peracids are able to inhibit as well bacterial protein tyrosine phosphatase, $\mathrm{YopH}$, with an $\mathrm{IC}_{50}$ value of $41 \mathrm{nM}$ observed for C8-peracid (data not shown), after 15 minutes incubation time in recombinant YopH activity assay. We observed that peracids also decreases the activity of CD45 phosphatase natively present in the Jurkat cell line (Fig. 3A). It is worth mentioning, however, that peracids have limited effect on Jurkat cell viability, comparing to hydrogen peroxide (Fig. 3B). Significantly higher impact of hydrogen peroxide on cell viability may be due to the fact that hydrogen peroxide can easily cross the cell membrane and cause damage to cellular biomolecules either directly or after conversion, in presence of metal ions, to more reactive hydroxyl radical [45].

Interestingly, the obtained results point to a unique correlation between the length of the peracid hydrocarbon chain and its inhibitory effect on PTPs. Medium-chain peracids (containing 8-12 carbons in an acyl chain) proved to have a higher impact on PTPs inactivation than C2- or longer-chain peracids (Fig. 4). According to the docking studies, the mediumchain C8-, C9- and C10- peracids were predicted to bind with the strongest affinity to the CD45 active site, with the maximum binding affinity calculated for the C10-peracid (Fig. $5 A)$. These data are in agreement with the experimental results showing that medium-chain peracids have the strongest inhibitory effect on PTPs, as observed for both the recombinant protein and cell culture experiments (Fig. 3A,4A). The stronger inhibitory effect of peracetic acid in Jurkat cells in comparison to recombinant enzymes is likely due to the specific ability of peracetic acid to penetrate through the cell membrane. Peracetic acid is more lipid soluble and not susceptible to decomposition by catalase and peroxidase, which effectively neutralize the action of hydrogen peroxide [46]. Molecular dynamics simulations reveal no affinity of the C4-peracids (Fig. 6B) (C4 and $\mathrm{C}^{1-}{ }^{-}$form), for the $\mathrm{CD} 45$ active site, which explains the lack of inhibitory activity observed for the short-chain peracid (Fig. 4). The binding free energies obtained from these calculations, involving CD45 and selected peracids, demonstrate the C8-peracid has the highest affinity for the CD45 active site, with large binding contributions from Arg859 and Gln897 (Fig. 6C). The cationic Arg859 has a large electrostatic contribution when interacting with anionic peracids. The importance of this residue is supported by other work that found Arg859 is essential for catalytic activity of CD45 phosphatase because it plays a crucial role in substrate binding and in the process of catalysis [47]. Furthermore, the anionic peracids are mainly stronger binders to the CD45 active site than their neutral counterparts, indicating this may be their active form (Fig. 6B,C).

PTPs show a high similarity of the active site structural architecture, in contrast to the electrostatic surface potential [24]. It may be assumed that the differences observed in a level of inactivation of CD45, PTP1B and LAR by peracids and hydrogen peroxide (Fig. 4) could be involved in the various active site electrostatic surface potential. The mapping studies of PTPs surface determined only a few conserved surface patches in the vicinity of

\section{KARGER}


the active site, which may result in the variation of substrate specificity [23].

The experimental results demonstrated that the inactivation caused by the mediumchain peracids is virtually irreversible (Fig. 5B). The enzymatic activity of CD45 inactivated by treatment with medium-chain peracids may only be restored by a few percent relative to the control. Conversely, the treatment of CD45 phosphatase with short-chain or longchain peracids, as well as hydrogen peroxide, induces reversible rather than irreversible oxidation, and the enzymatic activity is almost completely recovered with DTT (Fig. 5B). Previous studies indicated that organic peroxides, such as peracetic acid, induce reversible inactivation of PTP1B phosphatase [21]. According to Bhattacharya et al. [21], reversible PTP oxidation may be a general mechanism of enzymatic inactivation by organic peroxides. The results of this study clearly show that the reversible oxidation is not observed for the medium-chain peracids, possibly because their optimal size and structure allows for effective penetration into the catalytic center of CD45 repeatedly, leading to further oxidation of the catalytic cysteine to the irreversible sulfinic and sulfonic acid derivatives.

The highly-selective inactivation of PTPs by peracids correlates with hydrocarbon chain length, revealing medium-chain peracids, specifically peroctanoic acid (C8-peracid), to be the most effective oxidizing inhibitors of PTPs. Moreover, our experimental data suggest that inactivation of CD45 by medium-chain peracids is irreversible and leads to a decrease in a number of thiol groups, which are located on cysteine residues (Fig. 5C). Computational analysis showed that there are few structural changes after binding of peroctanoic acid within the CD45 active site (Fig. 6D), with the exception of the side chain of Asp821, His822 and Gly823, which are located near the active site.

The mechanism of CD 45 inactivation caused by peracids is still unclear, but we suspect it may occur through the irreversible oxidation of the cysteine residue, located in the catalytic center, to forms of sulfinic and sulfonic acid. Further studies on the mechanism of inactivation of PTPs by peracids are underway. However, because of the high reactivity of peracids, they are unlikely to accumulate in the cell and, therefore, the amount of peracids is hard to measure under in vivo conditions. Unfortunately, due to the highly-explosive properties of short-chain peracids, C4- and C6-peracids have not been synthetized and tested.

PTPs are potential pharmacological targets for novel drugs being developed in order to treat numerous pathologies including cancer, autoimmune disorders, allergic response, cardiovascular or neurodegenerative diseases $[48,49]$. Moreover, since many PTPs finetune subtle regulation of microbial biochemistry controlling the viability and virulence, they can be candidates for new therapies of infection diseases $[16,50]$. The studies on inhibitory properties of peracids against PTPs may be a good starting point for the synthesis of analogous inhibitors characterized by higher selectivity and stability, potentially leading to clinical application. In conclusion, the obtained data suggest that peracids are potent inhibitors of PTPs with the strongest inhibitory effect observed for medium-chain peracids, which correlate with an optimal binding affinity.

\section{Acknowledgements}

This work was supported by Grant No. 2012/07/N/NZ1/00012 from Polish National Science Center. JAT gratefully acknowledges support from Natural Sciences and Engineering Research Council of Canada (NSERC), the Canadian Breast Cancer Foundation - Prairies/ NWT Region and the Allard Foundation. MK gratefully acknowledges the support from NSERC. CDMC thanks NSERC, Alberta Innovates - Technology Futures and University of Alberta for student scholarships. Computations were performed using WestGrid resources. MW acknowledges Medical University of Gdansk Funding No. ST46.

\section{Disclosure Statement}

The authors declare no competing financial interest, or other interests that might be perceived to influence the results and/or discussion reported in this article. 


\section{Cellular Physiology Cell Physiol Biochem 2015;36:1069-1083 \begin{tabular}{l|l}
\hline DOI: 10.1159/000430280 & (c) 2015 S. Karger AG, Basel
\end{tabular}

Kuban-Jankowska et al.: PTPs as Targets for Peracids

\section{References}

1 Tonks NK: Protein tyrosine phosphatases: from genes, to function, to disease. Nat Rev Mol Cell Biol 2006;7:833-846.

2 Tabernero L, Aricescu AR, Jones EY, Szedlacsek SE: Protein tyrosine phosphatases: structure-function relationships. FEBS J 2008;275:867-882.

3 Pagliarini DJ, Robinson FL, Dixon JE: Protein Tyrosine Phosphatases; in Encyclopedia of Biological Chemistry. San Diego, Elsevier Inc, 2004, vol 3, pp 536-542.

4 Ostman A, Frijhoff J, Sandin A, Bohmer F: Regulation of protein tyrosine phosphatases by reversible oxidation. J Biochem 2011;150:345-356.

5 Persson C, Sjöblom T, Groen A, Kappert K, Engström U, Hellman U, Heldin CH, den Hertog J, Ostman A: Preferential oxidation of second phosphatase domain of receptor-like PTP- $\alpha$ revealed by an antibody against oxidized protein tyrosine phosphatases. Proc Natl Acad Sci 2004;101:1886-1891.

6 Hermiston ML, Zikherman J, Zhu JW: CD45, CD148, and Lyp/Pep: Critical phosphatases regulating Src family kinase signaling networks in immune cells. Immunol Rev 2009;228:288-311.

7 Tan J, Town T, Mori T, Wu Y, Saxe M, Crawford F, Mullan M: CD45 opposes beta amyloid peptide-induced microglial activation via inhibition of p44/42 mitogen-activated protein kinase. J Neurosci 2000;20:75877594.

8 Dios ID, Ramudo L, Alonso JR, Recio JS, Garcia-Montero AC, Manso MA: CD45 expression on rat acinar cells: Involvment in pro-inflammatory cytokine production. FEBS Lett 2005;579:6355-6360.

9 Huntington ND, Tarlington DM: CD45: direct and indirect government of immune regulation. Immunol Lett 2004;94:167-174.

10 Um JW, Ko J: LAR-RPTPs: synaptic adhesion molecules that shape synapse development. Trends Cell Biol 2013;23:465-475.

11 Ajay D, Sobhia ME: Identification of novel, less toxic PTP-LAR inhibitors using in silico strategies: pharmacophore modeling, SADMET-based virtual screening and docking. J Mol Model 2012;18:187-201.

12 Zhang S, Zhang ZY: PTP1B as a drug target: recent developments in PTP1B inhibitor discovery. Drug Discov Today 2007;12:373-381.

13 Bence KK, Delibegovic M, Xue B, Gorgun CZ, Hotamisligil GS, Neel BG, Kahn BB: Neuronal PTP1B regulates body weight, adiposity and leptin action. Nat Med 2006;12:917-924.

14 Aceto N, Bentires-Alj M: Targeting protein-tyrosine phosphatases in breast cancer. Oncotarget 2012;3:514515.

15 Lessard L, Stuible M, Tremblay ML: The two faces of PTP1B in cancer. Biochim Biophys Acta 2010;1804:613-619.

16 Bohmer F, Szedlacsek S, Tabernero L, Ostman A, den Hertog J: Protein tyrosine phosphatases structurefunction relationships in regulation and pathogenesis. FEBS J 2012;280:413-431.

17 Trosky JE, Liverman ADB, Orth K: Yersinia outer proteins: Yops. Cell Microbiol 2008;10:557-565.

18 Rubio M, Ramirez-Galicia G, Lopez-Nava LJ: Mechanism formation of peracids. J Mol Struct (Teochem) 2005;726:261-269.

19 Klenk H, Gotz PH, Siegmeier R, Mayr W: Peroxy compounds, organic. Weinheim, Wiley VCH Verlage GmbH \& Co, 2012.

20 Allen DW, Newman LM, Okazaki IJ: Inhibition of arachidonic acid incorporation into erythrocyte phospholipids by peracetic acid and other peroxides. Role of arachidonoyl-CoA: 1-palmitoyl-snglycero-3phosphocholine acyl transferase. Biochem Biophys Acta 1991;1081:267-273.

21 Bhattacharya S, LaButti JN, Seiner DR, Gates KS: Oxidative inactivation of protein tyrosine phosphatase 1B by organic hydroperoxides. Bioorg Med Chem Lett 2008;18:5856-5859.

22 Kuban-Jankowska A, Tuszynski JA, Winter P, Gorska M, Knap N, Wozniak M: Activation of hydrogen peroxide to peroxytetradecanoic acid is responsible for potent inhibition of protein tyrosine phosphatase CD45. PLoS One 2012; 7:e52495.

23 He Y, Zeng LF, Yu ZH, He R, Liu S, Zhang ZY: Bicyclic benzofuran and indole-based salicylic acids as protein tyrosine phosphatase inhibitors. Bioorg Med Chem 2012;20:1940-1946.

24 Barr AJ, Ugochukwu E, Lee WH, King ON, Filippakopoulos P, Alfano I, Savitsky P, Burgess-Brown NA, Müller S, Knapp S: Large-scale structural analysis of the human protein tyrosine phosphatome. Cell 2009;136:352363.

25 Parker WE, Ricciuti C, Ogg CL, Swern D: Peroxides II. Preparation, characterisation and polarographic behaviour of long-chain aliphatic peracids. J Am Chem Soc 1955;77:4037-4041. 


\section{Cellular Physiology Cell Physiol Biochem 2015;36:1069-1083 \begin{tabular}{l|l|l}
\hline DOI: 10.1159/000430280 & (C)15 S. Karger AG, Basel
\end{tabular} \\ Kuban-Jankowska et al.: PTPs as Targets for Peracids}

26 Morris GM, Huey R, Lindstrom W, Sanner MF, Belew RK, Goodsell DS, Olson AJ: AutoDock4 and AutodockTools4: automated docking with selective receptor flexibility. J Comput Chem 2009;16:27852791.

27 Trott 0, Olson AJ: AutoDock Vina: improving the speed and accuracy of docking with a new scoring function, efficient optimization and multithreading. J Comput Chem 2010;31:455-461.

28 Bialy L, Waldmann H: Inhibitors of protein tyrosine phosphatases: next-generation drugs? Angew Chem Int Ed Engl 2005;44:3814-3839.

29 Wang JM, Wolf RM, Caldwell JW, Kollman PA, Case DA: Development and testing of a general amber force field. J Comput Chem 2004;25:1157-1174.

30 Hornak V, Abel R, Okur A, Strockbine B, Roitberg A, Simmerling C: Comparison of multiple Amber force fields and development of improved protein backbone parameters. Proteins 2006;65:712-725.

31 Case DA, Darden TA, Cheatham III TE, Simmerling CL, Wang J, Duke RE, Luo R, Walker RC, Zhang W, Merz KM, Roberts B, Hayik S, Roitberg A, Seabra G, Swails J, Goetz AW, Kolossváry I, Wong KF, Paesani F, Vanicek J, Wolf RM, Liu J, Wu X, Brozell SR, Steinbrecher T, Gohlke H, Cai Q Ye X, Wang J, Hsieh MJ, Cui G, Roe DR, Mathews DH, Seetin MG, Salomon-Ferrer R, Sagui C, Babin V, Luchko T, Gusarov S, Kovalenko A, Kollman PA: AMBER 12; University of California. San Francisco, 2012.

32 Le Grand S, Götz AW, Walker RC: SPFP: Speed without compromise - a mixed precision model for GPU accelerated molecular dynamics simulations. Comput Phys Commun 2013;184:374-380.

33 Götz AW, Williamson MJ, Xu D, Poole D, Le Grand S, Walker RC: Routine Microsecond Molecular Dynamics Simulations with AMBER on GPUs. 1. Generalized Born. J Chem Theory Comput 2012;8:1542-1555.

34 Salomon-Ferrer R, Götz AW, Poole D, Le Grand S, Walker RC: Routine microsecond molecular dynamics simulations with AMBER on GPUs. 2. Explicit Solvent Particle Mesh Ewald. J Chem Theory Comput 2013;9:3878-3888.

35 Mongan J, Simmerling C, McCammon JA, Case DA, Onufriev A: Generalized Born model with a simple, robust molecular volume correction. J Chem Theory Comput 2007;3:156-169.

36 Nguyen H, Roe DR, Simmerling C: Improved Generalized Born Solvent Model Parameters for Protein Simulations. J Chem Theory Comput 2013;9:2020-2034.

37 Conrad M, Sandin A, Förster H, Seiler A, Frijhoff J, Dagnell M, Bornkamm GW, Rådmark O, Hooft van Huijsduijnen R, Aspenström P, Böhmer F, Ostman A: 12/15-lipoxygenase- derived lipid peroxides control receptor tyrosine kinase signaling through oxidation of protein tyrosine phosphatases. Proc Natl Acad Sci 2010;107:15774-15779.

38 Strukul G: Catalytic oxidations with hydrogen peroxide as oxidant. Dordrecht, Kluwer Academic Publisher, 1992.

39 Bjorkling F, Frykman H, Godtfredsen SE, Kirk O: Lipase Catalysed Synthesis of Peroxycarboxylic Acids and Lipase Mediated Oxidations. Tetrahedron 1992;48:4587-4592.

40 Glasgow JF, Middleton B: Reye syndrome- insights on causiation and prognosis. Arch Dis Child 2001;85:351-353.

41 Khyshiktuev BS, Karavaeva TM, Fal'ko EV: Variability of quantitative changes in short- chain fatty acids in serum and epidermis in psoriasis. Klin Lab Diagn 2008;8:22-24.

42 Meng TC, Buckley DA, Galic S, Tiganis T, Tonks NK: Regulation of insulin signaling through reversible oxidation of the protein-tyrosine phosphatases TC45 and PTP1B. J Biol Chem 2004;279:37716-37725.

43 Shibata E, Kanno T, Tsuchiya A, Kuribayashi K, Tabata C, Nakano T, Nishizaki T: Free fatty acids inhibit protein tyrosine phosphatase 1B and activate Akt. Cell Physiol Biochem 2013;32:871-879.

44 Liu D, Kong G, Chen QC, Wang G, Li J, Xu Y, lin T, Tian Y, Zhang X, Yao X, Feng G, Lu Z, Chen H: Fatty acids as natural specific inhibitors of the proto-oncogenic protein Shp2. Bioorg Med Chem Lett 2011;21:6833-6837.

45 Halliwell B, Clement LV, Long LH: Hydrogen peroxide in the human body. FEBS Lett 2000;486:10-13.

46 Klopotek BB: Peracetic acid methods for preparation and properties. Chimica Oggi 1998;16:33-37.

47 Kolmodin K, Aqvist J: The catalytic mechanism of protein tyrosine phosphatases revisited. FEBS Lett 2001;498:208-213.

48 Alonso A, Sasin J, Bottini I, Friedberg I, Osterman A, Godzik A, Hunter T, Dixon J, Mustelin T: Protein tyrosine phosphatases in the human genome. Cell 2004;177:699-711.

49 Hendriks WJAJ, Elson A, Harroch S, Stoker AW: Protein tyrosine phosphatases: functional inferences from mouse models and human diseases. FEBS J 2008;275:816-830.

50 Kuban-Jankowska A, Gorska M, Knap N, Cappello F, Wozniak M: Protein tyrosine phosphatases in pathological process. Front Biosci (Landmark Ed) 2015;20:377-388. 